\title{
Quaderni
}

QUADERNI Communication, technologies, pouvoir

92 | Hiver 2016-2017

Les artistes à l'école : fin d'une illusion ou utopie en devenir?

\section{Faire ensemble. Enjeux du partage du travail éducatif en contexte d'éducation artistique}

Jean-Paul Filiod

\section{(2) OpenEdition}

Journals

Édition électronique

URL : http://journals.openedition.org/quaderni/1037

DOI : 10.4000 /quaderni. 1037

ISSN : 2105-2956

Éditeur

Les éditions de la Maison des sciences de l'Homme

Édition imprimée

Date de publication : 5 mars 2017

Pagination : 49-61

Référence électronique

Jean-Paul Filiod, « Faire ensemble. Enjeux du partage du travail éducatif en contexte d'éducation artistique », Quaderni [En ligne], 92 | Hiver 2016-2017, mis en ligne le 05 mars 2019, consulté le 03 janvier 2020. URL : http://journals.openedition.org/quaderni/1037 ; DOI : 10.4000/quaderni.1037

Tous droits réservés 


\section{$D$ ossier}

\section{Faire ensemble. Enjeux}

du partage du travail éducatif

en contexte d'éducation artistique

Jean-Paul Filiod

Sociologue anthropologue Université Claude Bernard Lyon 1 (CESPÉ), Centre Max Weber
L'expression «Éducation artistique et culturelle » est consacrée depuis plusieurs décennies en France par des politiques publiques qui attestent d'un maillage entre les ministères de la Culture et de l'Éducation nationale, et qui ont donné lieu, entre autres, au Plan pour les arts et la culture à l'École et au Parcours d'éducation artistique et culturelle ${ }^{1}$. Les célébrations qui ont entouré l'entrée au Panthéon de Jean Zay, le 27 mai 2015, ont rappelé l'importance de cette ouverture vers les arts et la culture dans l'éducation, qu'a initiée ce jeune ministre à la fin des années 1930. Depuis cette période jusqu'à nos jours, les politiques publiques ont homologué avec constance la formule éducation artistique et culturelle, domaine généralement considéré comme relevant de l'innovation ${ }^{2}$. Nous préférerons ici parler d'éducation artistique. D'une part, pour éviter de rattacher la culture systématiquement à l'art, alors qu'elle est plus générale : elle sollicite les comportements les plus quotidiens, structure des modes de vie et imprègne aussi le domaine des sciences et des techniques ( $c f$. la culture scientifique et technique). D'autre part, cela permet de recentrer l'attention sur l'art, les arts et les artistes, alors mobilisés dans des contextes différents de ceux d'un « marché de l'Art » qui les rattache à des lieux spécifiques (galeries, musées, salles de spectacle).

L'éducation artistique comporte trois formes cognitives, reconnues tant par les chercheurs ${ }^{3}$ que par les politiques publiques ${ }^{4}$ :

- rencontrer des œuvres, apprendre à les connaître, grâce à l'activité perceptive et sensorielle ;

- réfléchir sur des œuvres, les comprendre, apprécier les intentions de leurs auteurs ; 
- produire des œuvres, travailler la matière, faire travailler les sens.

Les projets ou partenariats d' " éducation artistique et culturelle » accordent plus ou moins de poids à telle ou telle de ces formes cognitives. Le « culturel » renvoyant surtout à de la connaissance, certains acteurs pensent qu'il convient d'insister sur cet aspect ; d'autres, appuyant plus sur l'« artistique », défendent la contribution d'un(e) artiste, que ses connaissances et compétences soient mises à profit pour une «médiation artistique et culturelle » (lors de biennales, festivals, expositions) ou pour une pratique artistique avec des enfants ou des adolescents. Nous nous intéresserons ici aux situations en milieu scolaire où est valorisée la troisième option, en nous appuyant sur une longue expérience de terrain sur le « programme de résidences d'artistes en école maternelle » Enfance Art et Langages (EAL), que la Ville de Lyon a créé en 2002, sous le premier mandat de Gérard Collomb, et supprimé au début de son troisième, en $2015^{5}$.

Entre 2004 et 2015, ces recherches ont permis d'observer et d'analyser nombre de situations mettant en jeu des enseignants, des artistes et des Atsem (agent territorial spécialisé des écoles maternelles). L'attribution d'un nom à ces métiers engendre une représentation de ce que ces personnes font, sont censées faire, ou de ce qu'on attend qu'elles fassent : l'enseignant enseigne, l'artiste crée, l'Atsem assiste l'enseignant (ménage, soin aux enfants, préparation matérielle des activités). Pourtant, le terrain renvoie autre chose que cette image faisant coïncider strictement un groupe professionnel, un statut, des fonctions et des rôles. Profuses sont les situations et occasions de transmission et de médiation, variés sont les contextes des écoles concernées (de sept à dix par an), cette profusion et variété agissant fortement sur les rôles des uns et des autres.

Nous allons donc examiner de plus près cette tension entre la prescription de fonctions propre à chacun des métiers et les variations contextuelles et individuelles. Un détour par la sociologie des professions permettra de faire émerger les enjeux $\mathrm{du}$ faire-ensemble, que nous mettrons en perspective vis-à-vis de la logique de "partenariat », qui relève de l'injonction institutionnelle autant qu'elle met les acteurs face à des expériences singulières et souvent inédites. Des formes de collaboration émergent, susceptibles de s'offrir comme « innovations ». En étayant notre propos par des exemples tirés du terrain EAL, nous verrons comment ce qui est innovation pour certains ne l'est pas toujours pour d'autres ; s'impose alors une interrogation sur la réflexivité partagée au sein de collectifs partenariaux dans lesquels les chercheurs ont sans doute un rôle à jouer.

\section{Des groupes professionnels face au travail éducatif}

La critique des sociologues interactionnistes anglo-saxons à l'encontre de leurs homologues fonctionnalistes entre 1930 et $1950^{6}$ a acté le fait que le mot profession ne devait pas être réservé aux métiers à « haut niveau de savoir» : les «petits métiers » (occupation) procèdent eux aussi de savoirs complexes. En France, une « sociologie des groupes professionnels » s'est constituée, qui considère alors tout métier comme une profession, au-delà de la hiérarchisation des qualifications. Tous les métiers sont donc à 
prendre au sérieux, y compris ceux dont on pense qu'ils sont limités à une fonction d'exécution, par exemple, ici, les Atsem.

Raisonner par l'identité nominale pose toutefois problème. D'abord, si la profession désigne l'ensemble des personnes ayant le même métier ou statut (« médecins », " enseignants», « ingénieurs », « couvreurs »...), elle désigne aussi l'ensemble des travailleurs d'un même secteur d'activités ayant une «profession» différente (" professionnels de l'habitat», « de la santé », « de l'informatique », « de la petite enfance $» . .$.$) . Mais surtout, une définition par$ l'identité nominale présente le risque de ne nous faire voir qu'une substance, quand la réalité montre des fluctuations, du fait d'interactions entre les membres de ces « groupes » et d'autres acteurs : «Pour étudier correctement la division du travail, il faut, dans chaque système de travail, prendre en compte le point de vue de toutes les catégories de personnes qui y sont impliquées, que leur position soit supérieure ou inférieure, qu'ils soient au centre ou à la périphérie du système. ${ }^{7}$ " Si le trio enseignant-artiste-Atsem fait système dans le travail au quotidien, alors nous devons à la fois déhiérarchiser ces groupes professionnels et prêter attention aux changements en leur sein, comme au sein du système dans lequel ils évoluent. Interroger la professionnalité en contexte - contexte éducatif en la circonstance-demande de ne pas considérer l'éducation comme un simple corpus idéologique servi par des discours mais comme une activité : tout adulte en présence d'enfants-élèves ${ }^{8}$ fait a minima un « travail éducatif »?.

Certains, comme les enseignants, sont toute- fois appelés "professionnels de l'éducation ", quand le terme " éducateurs » est réservé aux « travailleurs sociaux ». Mais comme l'éducation relève d'une conception large ayant trait aux valeurs, aux normes, aux règles de base qui distribuent les comportements selon des prescriptions, des proscriptions et des tolérances, certains préfèrent voir les enseignants comme des «professionnels de l'enseignement » ou « de la pédagogie ». En même temps, quand l'enseignant travaille en école maternelle, on ne le désigne pas comme un " professionnel de la petite enfance », catégorie à laquelle appartiennent les Atsem (et autres éducateurs de jeunes enfants [EJE], puéricultrices et assistantes maternelles). L'entrée par le travail éducatif trouble ainsi les catégories et rend plus complexe (mais peut-être plus intéressante) l'analyse des partenariats d'éducation artistique.

Au-delà de ces enjeux de définition, la sociologie et l'anthropologie prennent en compte les dimensions objectives et subjectives des phénomènes qu'elles étudient, constatant à la fois les changements survenus dans les groupes professionnels et la singularité des parcours et des formes d'engagement des acteurs. Ainsi les enseignants, soumis à l'hétérogénéité des publics scolaires et à la perte du monopole de la culture (phénomène plus général d'une « fin des monopoles » qui touche les institutions ${ }^{10}$ ), sont-ils confrontés à une pluralité de modèles. Par exemple, Monique Hirschhorn en dégage cinq (magister, pédagogue, animateur, formaliste ou utilitariste ${ }^{11}$ ), et Léopold Paquay, six (maître instruit, technicien, praticien-artisan, praticien réflexif, acteur social ou personne ${ }^{12}$ ). De nos jours, l'enseignant, membre d'un groupe professionnel peu homogène, fait face en classe 
à « un multi-agenda de préoccupations enchâssées $»^{13}$ et en classe comme au-delà d'elle à du 《 travail partagé $»^{14}$.

Dans les partenariats d'éducation artistique, ces mêmes enseignants, dotés d'une formation institutionnelle sur l'éducation et la pédagogie, sont amenés à travailler avec des acteurs qui n'en ont pas ou peu : les artistes ${ }^{15}$. Les recherches sur le travail artistique, depuis les travaux pionniers de H. S. Becker ${ }^{16}$, étudient le métier d'artiste à partir de ses réalités concrètes et ses formes collectives. Nous poursuivons ici la mise au jour à laquelle ces travaux invitent et qui nous aident à évacuer l'image simpliste d'artistes déterminés par la seule inspiration. Les résidences EAL furent suffisamment consistantes en espaces-temps pour constater que les artistes travaillant là activaient des compétences éducatives, voire pédagogiques. L'usage de l'index pour montrer, démontrer, expliquer, ne fut pas rare, comme le fait de le placer devant la bouche en disant « Chhhtt» pour obtenir le silence. De même : produire un énoncé en début d'activité en vue d'un apprentissage précis, faire parler les enfants-élèves sur le mode question-réponse pour engager la construction de connaissances, voire, même si ce fut plus rarement constaté, faire dire les bonnes réponses ou afficher des règles de vie commune dans l'espace commun, deux pratiques qui relèvent de ce que font les enseignants. Ces pratiques, apprises par les artistes dans l'implicite de leur propre éducation ou lors d'une formation (d'animateur, par exemple) ou empruntées aux enseignants ou aux Atsem fréquentés, ont ainsi partie liée avec les formes culturelles de l'institution scolaire. Des différences existent, cependant, entre la pratique éducative de l'enseignant et celle de l'artiste.
On note par exemple l'insistance avec laquelle certains artistes parlent de « proposition » pour définir « l'énoncé initial de l'activité », quand les enseignants privilégient le mot $«$ consigne $»^{17}$. Importe alors ce qui se joue dans l'écart à l'énoncé initial de l'activité, plutôt qu'une adéquation entre l'objectif sous-tendu par la consigne et les résultats obtenus ${ }^{18}$. Du point de vue des genres pédagogiques, il y a là une proximité avec les pédagogies de la découverte plutôt qu'avec celles de la transmission.

Rien de tel chez les Atsem, dont on n'attend pas de compétence proprement pédagogique. La compétence éducative de ces ex-« femmes de service » est en revanche signifiée depuis 1992, par l'exigence d'un CAP petite enfance. Dès lors, l'une des questions est la reconnaissance de cette dimension éducative dans le métier, qui figure une réalité récente pour ce personnel dont les missions d'entretien des locaux et des enfants (hygiène, propreté, sécurité) restent malgré tout dominantes dans les pratiques et les attentes sociales, et ce, y compris chez les enseignants d'école maternelle. Certains d'entre eux considèrent en effet l'Atsem comme un(e) assistant(e) qui sera informé au dernier moment de l'activité qu'il a planifiée. D'autres, en revanche, reconnaissent aux Atsem une place éducative au point de les impliquer dans des activités pédagogiques et de les informer par avance. Bien sûr, certain(e)s Atsem, fort(e)s de leur formation et de leur qualification, s'impliquent d'elles-mêmes, quand d'autres se limitent au minimum, considérant que la quantité de travail à produire est déjà importante et que le salaire ne suit pas ${ }^{19}$. Dans tous les cas, le jeu d'interactions entre l'enseignant et l'Atsem produit une relation spécifique, susceptible de 
changer au fil du temps. La définition structurelle de la subordination à l'enseignant peut donc être atténuée en certains endroits, voire effacée en cas de recherche mutuelle de symétrie dans l'activité professionnelle.

Cette nouvelle donne professionnelle, couplée au contexte d'éducation artistique, a donné à certaines Atsem une impulsion particulière, comme le montre 1'exemple suivant. Cette Atsem, qui travaillait dans une école accueillant une plasticienne en résidence, a appris, en formation, qu'en maternelle, on explore, et même si «ça fout le bazar », on sait que c'est pour le bien de l'enfant. Elle souligne ici l'importance des interactions entre les comportements des enfants et les lieux dans lesquels ils évoluent : "Pour que l'enfant puisse s'épanouir dans un endroit, il faut qu'il puisse retrouver l'endroit comme il l'a trouvé la veille, dans un état de propreté irréprochable. [...] Et je sais que les vitres, quand ils se regardent et qu'ils voient des traces, moi je l'ai vu plusieurs fois quand ils sont à l'extérieur, quand il y a des traînées, ils n'osent plus se mettre contre. Alors pourquoi?, j'en sais rien, mais quand de nouveau c'est nickel, faites l'expérience, vous verrez, ils mettront leur visage, ils mettront leurs lèvres, leur nez et puis ils s'écraseront dessus. [...] Y a un miroir... Écoutez, je peux vous parier que si le miroir, on le fait pas, l'enfant n'ira pas se faire des grimaces et passera à côté. » Faire le ménage, ici, on le voit, n'est pas qu'un acte de remise en ordre des choses. L'acte de nettoyage des lieux et l'activité des enfants-élèves s'imbriquent, à partir d'une conscience de l'importance de l'acte d'observer, jusque dans les plis du quotidien.

Alors oui, bien sûr, l'enseignant enseigne,
l'Atsem l'assiste et l'artiste crée. Mais dans ce contexte à la fois scolaire et partenarial centré sur l'éducation artistique, les positions bougent, les situations impliquent les sujets professionnels dans des actes différents qui les impliquent dans des négociations et des ajustements réciproques.

\section{Partenariats et projets : l'injonction et l'expérience}

Le mot partenariat est arrivé dans le vocabulaire des entreprises, du système éducatif et des dictionnaires dans les années $1980^{20}$. Le terme relève de «l'injonction institutionnelle à "faire ensemble" " et signifie moins la «légitimation de pratiques existantes " que la justification d'une " "gouvernance" scolaire » résultant d'une « complexification des dispositifs éducatifs nécessitant le concours d'acteurs les plus divers $^{21} »$. Si la compréhension des partenariats en terme de gouvernance est utile, rendant compte de la logique dominante des politiques publiques (l'évaluation et le new public management ${ }^{22}$ ), elle risque de laisser de côté la manière dont se déroulent et se vivent les partenariats. D'autant que les acteurs des partenariats sont appelés partenaires, terme ancien (vers 1780) qui renvoie à des relations plus interindividuelles qu'institutionnelles : on en use pour parler du jeu, du théâtre, du cinéma, de la danse, de la sexualité.

En nous situant à cette échelle de l'école au quotidien, on peut distinguer trois types d'acteurs, en partant d'un ego défini par son appartenance à un groupe professionnel :

- ceux qui appartiennent au même groupe que cet ego : les mêmes;

- ceux qui n'y appartiennent pas mais travaillent 
au quotidien avec ego : les proches;

- ceux qui n'y appartiennent pas et ne travaillent qu'occasionnellement avec ego : les autres.

Pour l'enseignant, l'Atsem fait partie des proches, l'artiste, des autres, comme pour l'Atsem. Globalement, l'altérité peut s'entendre au sein d'un groupe de mêmes ou de proches ou entre mêmes et proches. Mais elle suppose aussi un écart significatif en terme de différence et un minimum d'obligation de faire avec l'autre. Ce qui sous-entend des contacts répétés, l'entrée dans une relation ${ }^{23}$ qui, petit à petit, fait histoire, l'altérité devient processus ${ }^{24}$. Dans la rencontre, les normes et les codes portés par chacun se soumettent à de potentielles inflexions, du fait de l'existence d'une rencontre : « Connaître, c'est se familiariser avec quelqu'un par approximations, approches successives et jeu de conjectures, et non le soumettre à un savoir codifié..$^{25} \gg$ La découverte progressive, dès lors qu'elle comporte de la réciprocité, pose la question de la transformation de soi par l'autre et de l'autre par soi. Or les projets et partenariats ont bien cette caractéristique de créer des formes de coopération et de collaboration qui s'écartent de la logique des identités professionnelles et du travail quotidien entretenu par des habitudes. L'exemple des artistes donne souvent lieu à des constats sur un grand écart altéritaire $^{26}$, ce qui pose souvent problème dans le contexte institutionnel de l'École, dont l'unité signifiante est souvent présentée comme menacée (l'image du « sanctuaire » y est régulièrement appliquée depuis les années 1990, les protections supplémentaires, physiques ou télécommandées, n'y sont pas rares). Mais cela ne l'empêche pas de bouger de l'intérieur, qu'on parle de l'organisation, des pratiques ou des sujets individuels et collectifs.

Ainsi, une enseignante prend des photos pendant qu'une chorégraphe dirige son groupe d'enfants-élèves, tandis qu'une Atsem entre dans la danse. Puis l'Atsem prend l'appareil photo, l'enseignante se met en retrait, observe, puis se met elle aussi à danser. L'activité achevée, chacun raccompagne le groupe dans la classe, avec les mêmes rôles : inciter les enfants-élèves à se chausser, se déplacer sans encombre et sans trop de dissipation. Cet exemple sous-entend toutefois que les trois adultes sont ensemble avec la totalité des enfants-élèves. Or, la plupart du temps, les enfants-élèves sont en sous-groupes, une demi-classe ou jusqu'à des groupes de quatre enfants-élèves. Ces possibilités sont plus facilement offertes en école maternelle, du fait de l'existence des Atsem : la présence de trois adultes permet de diversifier les activités selon les objectifs visés. Et de bonnes raisons existent dans ce choix de ne pas travailler avec la classe entière : des échanges plus individualisés, un travail plus aisé à mettre en place sur les langages et leur expression, une plus grande proximité avec l'artiste, aidant à construire une véritable "relation ${ }^{27}$. Dans certaines écoles, un choix plus radical a été fait : les enseignantes n'assistaient pas aux activités avec l'artiste, bien que l'inspecteur de circonscription, leur supérieur hiérarchique, ait dit l'intérêt qu'il y aurait pour elles de «s'impliquer corporellement ». Ce choix tenait au fait que les enfants-élèves " $s$ 'autorisent d'autres choses quand l'enseignante n'est pas là ", cette absence présentant un autre avantage : n'ayant rien vu de la séance, l'enseignante s 'offrait en candide à son groupe, obligé d 'exprimer verbalement les activités réalisées avec l'artiste. 
On travaille ainsi l'expression, le vocabulaire, la taille du groupe permettant une durée plus grande d'expression pour chacun.

Les configurations organisationnelles ont varié d 'une école à l'autre, voire d'une année à l'autre au sein d'une même école. Ces variations proviennent de l'expérience, des tentatives et de leurs évaluations en local, mais aussi des nombreux échanges d'idées qui ont eu cours dans les lieux de formation (stages et séminaires de durée variable selon les époques) mis en place par Enfance Art et Langages. Dans ce partenariat, comme dans tant d'autres, les interactions ont produit des connaissances, des controverses, des questionnements sur les pratiques.

Cette réalité entérine l'impossibilité du « programme », au sens strict du mot. Dans le programma, la lettre précède l'action, qui devra donc suivre la lettre (" à la lettre »). Le programme n'est en effet qu'une forme particulière du pro-jet : ce qui est jeté avant l'action, ce -jet portant en lui un espace-temps du futur, plus ou moins proche. Tout ceci relève du pré-visionnel, de ce qu'on est susceptible de voir de ce futur. Alors, si le projet (n') est vu (que) comme un programme, il reste peu de place pour la prise en compte d'une conscience partagée de ce qu'il devient et de ce qui advient, c'est-à-dire de cette part du réel portée par les inflexions, les ajustements, régulations et autres négociations. Dans le cadre d'un projet pensé comme un programme, les négociations peuvent être perçues comme des freins ; or les projets et les partenariats sont faits de résistances, de dynamique inattendues, de critiques, de leviers insoupçonnés, de souffrances, de redistributions de rôles, voire de redéfinitions d'objectifs. Ils vivent ce que vit toute organisation, entre comportements affinitaires, fusionnels, de retrait ou de négociation ${ }^{28}$, du fait des différences de statut, de compétences ou de valeurs. Par ailleurs, le pro-jet, puisqu'il comporte des " su-jets », des « ob-jets », du " re-jet », un « tra-jet», revêt un caractère « existentiel $»^{29}$. Appréhender le projet par le trajet, par ce qui, littéralement, traverse ce qui est jeté, invite à une analyse processuelle et à la mise au jour de ses composantes : les ingrédients du contexte, les séquences, les moteurs et les bifurcations ${ }^{30}$. La tentation linéaire - ou plutôt ortholinéaire, la ligne des programmes aspirant plutôt à être droite - est mise à mal par la dynamique des projets et des partenariats, qui produit, ici ou là, quelques innovations. Et ce que donne à voir le travail de terrain et la démarche ethnographique, c'est que l'innovation n'est ni top-down (descendante) ni bottom-up (ascendante), mais plutôt, comme l'a montré la sociologie des innovations et de la traduction, «tourbillonnaire $»^{31}$.

\section{Paradoxes de l'innovation}

D'un point de vue général, l'attention aux sujets implique de prendre en compte, dans l'observation comme dans l'analyse, l'expérience de tous les sujets individuels et collectifs de l'innovation. Certains d'entre eux sont susceptibles d'innover, sans que ces nouvelles pratiques, dont le chercheur peut être un témoin privilégié, ne soient considérées comme des innovations par les institutions initiatrices du partenariat.

Les exemples de ce genre n'ont pas manqué sur le terrain des résidences d'artistes EAL. J'y ai relevé des changements de nature organisationnelle que 
je résume ici $^{32}$ :

- prise en charge des enfants-élèves pendant une demi-journée complète par l'artiste, parfois assisté d'une Atsem ;

- prise en charge d'un atelier d'une heure par une Atsem seule, une fois par semaine, le matin ; - présence de l'artiste dans d'autres espaces de l'école que le seul «atelier », «labo », «studio»; - réduction d'une heure du temps de ménage, pour permettre aux Atsem d'assister aux réunions sur les projets artistiques ;

- augmentation de la fréquence des temps de rencontre entre partenaires, y compris en dehors de l'école, dans des cadres publics ou privés ;

- mise à disposition d'un jeu de clefs de l'école pour que l'artiste puisse venir y travailler, y compris le week-end, ou y rester en dehors des heures de classe, l'école s'offrant comme résidence au sens fort.

Dans ces exemples, pas d'enseignants, mais leurs pratiques sont bien sûr affectées par ces changements. Par exemple lorsqu' on installe dans la classe un espace de création plastique parallèle à celui que l'artiste réalise dans les espaces collectifs de l'école. Ou lorsqu'on supprime, après une durée conséquente de la présence de l'artiste, certains « rituels » en « petite section » (première année de maternelle) «tels qu'ils se font habituellement, ça n'a pas de sens pour les enfants. [...] Ils ne doivent pas écouter la maîtresse qui dit “c'est lundi, gnagnagna”... ». Cette enseignante revendique toutefois un «rituel» central, «le déroulement de la matinée: avec des photos, on se dit ce qui va se passer le matin [...]. Si, plus tard, un enfant s'angoisse, je reviens sur cette bande de la matinée, et je lui dis "où on en est? qu' est-ce qu'il te reste?", il voit où il en est, on repart». Ces pratiques n'ont pas de mal à perdurer car la « liberté pédagogique » de l'enseignant s'applique, de même qu'il peut argumenter du bien-fondé de ses choix auprès de ses supérieurs (corps d'inspection), les programmes insistant depuis toujours sur le « percevoir», le « sentir », l'« imaginer ». En revanche, pour les changements organisationnels listés précédemment, c'est plus compliqué. Le maintien du cadre scolaire est le plus souvent une priorité et les responsables hiérarchiques peuvent effectuer un rappel à l'ordre, scolaire ou professionnel. Une relative inertie institutionnelle peut donc intervenir, comme le montre l'exemple suivant.

Une Atsem, qui se formait au massage shiatsu, a demandé à faire prendre en charge cette formation par sa hiérarchie. Elle refusa. L'Atsem décida alors de la payer sur ses propres deniers : « Moi, je l'avais dit avant, à mon inspecteur-coordinateur ${ }^{33}$ que s'il le prenait pas en compte, moi je le ferai quand même. Ça serait un plus pour moi et pour mes collègues, que ce soit enseignant, Atsem, je les masse, et quand y a des enfants ben, c'est par la respiration. [...] Moi je dis qu'on devrait accepter ces stages, ne serait-ce que pour apaiser. On a un enfant qui est hyperexcité dans la classe. Quand il l'est vraiment trop, moi je lui prends les mains, je lui demande de respirer calmement, d'inspirer, de souffler calmement, et au bout de... je sais pas, dix fois, il est beaucoup plus calme et il repart dans son activité. Si on laisse monter crescendo, il se retrouve, après, puni, c'est dommage ». L'artiste en résidence dans l'école n'était pas insensible à la question du toucher et fut enthousiaste à l'idée d'y travailler avec l'Atsem. Pendant l'année scolaire, à la fin de l'hiver, cette plasticienne revint d'un séjour au Mexique avec 
de nouveaux matériaux et de nouvelles manières de faire avec. Elle composa alors un document d'une trentaine de pages dans lequel elle installa un jeu de correspondances entre son expérience mexicaine et son travail dans l'école. Dans ce document, la question du toucher est explicite, rapportée à plusieurs réalités : la connaissance par le toucher ; le rapport entre sensibilité corporelle, potentialités de matériaux et invention de formes; et, au détour, une critique de la tendance au principe de précaution généralisé qui a cours en ce début de $\mathrm{XXI}^{\mathrm{e}}$ siècle.

L'artiste et l'Atsem ensemble, un assemblage porteur d'innovation? Une réunion de l'équipe éducative fera tourner court cette idée d'un travail collectif sur le toucher, impliquant la totalité des acteurs de l'école. On peut voir dans cette décision les effets d'une histoire du système éducatif français qui construit le rapport au savoir sur la base d'une distance corporelle minimale à respecter, selon l'idée qu'il se transmet d'esprit à esprit, de cerveau à cerveau. Mais est tout aussi déterminante la logique fonctionnaliste soutenue par les responsables hiérarchiques. Une inspectrice de l'Éducation nationale à qui je présentais cette situation n'a pas mis longtemps pour m'interrompre en disant net, à propos de l'Atsem masseuse : «Ce n'est pas dans ses missions. »

La situation peut donc devenir paradoxale. D'une part, le discours du partenariat enjoint les acteurs à ces déplacements (relationnels, personnels, professionnels), mais certains déplacements ne sont pas tolérés. D'autre part, l'Atsem masseuse offre une figure-limite intéressante : elle relève de la subversion vis-à-vis des normes d'intercorporéité adulte-enfant qui régissent l'École française, en même temps qu'elle confirme ce métier comme ayant le corps comme objet de travail et le care comme horizon professionnel. La subversion est toutefois relative, car ces exercices de mise en condition positive pour l'activité, notamment artistique, ne manquent pas d'être de plus en plus valorisés dans le système éducatif $f^{34}$.

\section{Conclusion}

La mise au jour, par le chercheur, de ces 《 innovations ordinaires $\|^{35}$ ou $~ «$ discrètes $\|^{36}$ les condamne-t-elle à le rester, dès lors que le chercheur n'a pas le pouvoir de décider de leur généralisation? Et quand il en fait part, s'agit-il de mettre en valeur ces innovations ou de mettre en valeur le fait qu'elles ont avorté ?

Dans cet article, il fut question des deux options pour montrer, d'une part, qu'il y a de l'innovation là où l'on ne s'y attend pas toujours, et d'autre part, que certaines formes du faire-ensemble ne sont pas toujours admises. Pourtant, le faireensemble a le vent en poupe. Le succès de l'économie dite " du partage » ou " collaborative » en atteste. Toutefois, les mondes du travail n'ont pas attendu qu'on parle de « collaboratif » pour produire du faire-ensemble, et l'attention au faireensemble ordinaire, quotidien, n'est pas nouvelle en sciences humaines et sociales, qui ont offert bien des occasions de réflexivité partagée dans ces mondes du travail. Mais partagée par qui ? et pour quoi faire?

La question est conséquente. La recherche menée dans le cadre d'EAL a permis d'animer des collectifs mêlant enseignants, Atsem et artistes. Cela est cependant resté limité : dans ce type de 
recherche, à tendance collaborative, il faudrait intégrer des inspecteurs, des formateurs, des personnels de direction, le chercheur jouant un rôle de traducteur pour traiter le sens des permanences et des changements qui affectent les objets mis en débat. Mais quand ces objets sont l'art, la pratique artistique, le travail artistique, l'éducation artistique, l'éducation tout court, voire le faire-ensemble lui-même, on sait combien se manifestent des passions et des jugements de valeur très soutenus. Les acteurs de la réflexivité partagée, sollicités pour discuter, voire valider, la pertinence de certaines innovations, tout ordinaires et discrètes qu'elles puissent être, devraient alors jouer le jeu de l'éthique, de la confiance également distribuée entre les partenaires, et se préserver de ce que les hiérarchies, les relations de subordination structurellement construites, sont susceptibles d'induire. Se mettre dans une telle disposition pourrait favoriser, au moins localement, des associations entre formes artistiques et pratiques éducatives, pour instiller dans l'école des changements pertinents du point de vue cognitif, culturel, professionnel et institutionnel. « Tout un programme »...

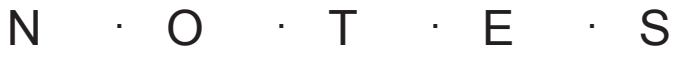

1. Protocole d'accord du 25 avril 1983, ministère de l'Éducation nationale, ministère délégué à la Culture. Protocole d'accord du 17 novembre 1993 relatif à l'éducation artistique, ministères de l'Éducation nationale, de la Culture et de la Francophonie, de l'Enseignement supérieur et de la Recherche, de la Jeunesse et des Sports. Le Plan pour les arts et la culture à l'École, Paris, CNDP, 2001. Circulaire interministérielle $\mathrm{n}^{\circ} 2008-059$ du 29 avril 2008 pour la mise en œuvre du Plan pour le développement de l'éducation artistique et culturelle. Le parcours d'éducation artistique et culturelle, BOEN n ${ }^{\circ}$ 19, 9 mai 2013. Parcours d'éducation artistique et culturelle, BOEN, $\mathrm{n}^{\circ} 28,9$ juillet 2015.

2. Jean Paul Filiod, «L'innovation en mode pluriel et relatif. L'exemple de l'éducation artistique et culturelle et d'un dispositif présenté comme "innovant" ", in Socio-logos. Revue de l'Association française de sociologie, $\mathrm{n}^{\circ} 7$ : L'innovation institutionnelle, 2012. socio-logos.revues.org/2661

3. Marie-Christine Bordeaux, «L'éducation artistique et culturelle », in G. Saez (éd.), Institutions et vie culturelles, Paris, La Documentation française, 2005, pp. 65-69. Howard Gardner, "Le 'Project Zero' de Harvard : trois leçons ", in Évaluer les effets de l'éducation artistique et culturelle, Paris, La Documentation française, 2008, pp. 69-73.

4. Éducation artistique. Développement de l'éducation artistique et culturelle, circulaire $n^{\circ} 2008-059 \mathrm{du}$ 29-4-2008, BOEN n 19 du 8 mai 2008. Le Parcours d'éducation artistique et culturelle, op. cit., 2015.

5. Sur cette suppression, $c f$. Jean Paul Filiod, «Arts et petite enfance au cœur d'une mésaventure démocratique », in AnthropoChildren, n $^{\circ}$ 6, 2016 (à paraître).

6. Florent Champy, Sociologie des professions, Paris, 
PUF, 2009, p. 16-31. Laure De Verdalle, « Professions », in A. Bevort, M. Lallement, A. Jobert, A. Mias (dir.), Dictionnaire du travail, Paris, PUF, 2012, pp. 569-575.

7. Everett C. Hughes, « Division du travail et rôle social ", in Le Regard sociologique, Paris, EHESS, 1996, p. 67. ( Social Role and the Division of Labor », in Midwest Sociologist, $\mathrm{n}^{\circ}$ 17(1), 1956, pp. 304-310.

8. Cette catégorie, que j'utilise depuis mes premières recherches sur EAL, permet d'intégrer le double statut, générationnel et scolaire, des êtres concernés. L'expression a aussi pour but de ne pas donner priorité à l'un des termes, cela pouvant être utilisé à des fins idéologiques : l'opposition entre les tenants de l'approche psychologique-pédagogique (enfant) et ceux de l'approche pédagogique-scolaire (élève), parfois brutale et stérile, ne nous est d'aucun secours.

9. Jean Paul Filiod, L'Éducation en partage. Une sociologie anthropologique du travail éducatif, mémoire d'habilitation à diriger des recherches, Université Claude Bernard Lyon 1, 2016.

10. François Dubet, Le Déclin de l'institution, Paris, Le Seuil, 2002, pp. 55-56.

11. Monique Hirschhorn, L'Ère des enseignants, Paris, PUF, 1993, chap. 5.

12. Léopold Paquay, «Vers un référentiel des compétences professionnelles de l'enseignant? », in Recherche et Formation, ${ }^{\circ} 16,1994$, pp. 7-38.

13. Dominique Bucheton, Yves Soulé, « Les gestes professionnels et le jeu des postures de l'enseignant dans la classe : un multi-agenda de préoccupations enchâssées ", in Éducation et Didactique, n 3, 2009/3, pp. 29-48.

14. Les Sciences de l'éducation. Pour l'ère nouvelle, $\mathrm{n}^{\circ} 42$ : Le travail partagé des enseignants, 2009/2.

15. Il existe des formations centrées sur la pédagogie et délivrant un diplôme universitaire : par exemple, les CFMI (centres de formation de musiciens intervenants, créés en 1984 par les ministères de la Culture et de l'Éducation nationale).

16. H. S. Becker, Art Worlds, University of California Press, 1982 ; trad. fr. Les Mondes de l'art, Paris, Flammarion, 1988.

17. Jean Paul Filiod, Sophie Necker, «Éducation artistique, évaluation et enjeux d'étiquetage. Une recherche collaborative en école dite "maternelle" ", in Interrogations?, $\mathrm{n}^{\circ} 22,2016$. revue-interrogations.org.

18. Ibid.

19. Le salaire des Atsem est inférieur à celui des enseignants et, dans le cadre d'EAL, le problème s'est posé aussi vis-à-vis des artistes. Lors des trois premières années de ce programme, les artistes, présents douze heures chaque semaine, recevaient des mensualités environ deux fois plus élevées que celles des Atsem. Des tensions résultaient parfois de ces écarts, sans que soit pris en compte le fait que les Atsem sont titulaires de la fonction publique, alors que les artistes étaient tenus chaque année à l'incertitude d'un renouvellement qui, en outre, ne pouvait se produire plus de deux fois. 20. Danielle Zay, "Partenariat ", in P. Champy, C. Étévé, Dictionnaire encyclopédique de l'éducation et de la formation, Paris, Nathan, 1994, p. 719-725. Corinne Merini, Christine de Peretti, «Partenariat externe et prévention en matière de substances psychoactives: dans quelle position l'école met-elle ses partenaires ? ", in Santé publique, n ${ }^{\circ}$ 14, 2002/2, pp. 147-164. Pascale Garnier, « Partenariats et réseaux éducatifs », in A. van Zanten (dir.), Dictionnaire de l'éducation, Paris, PUF, 2008, pp. 515-517.

21. P. Garnier, op. cit., p. 515.

22. Actes de la recherche en sciences sociales, $\mathrm{n}^{\circ} 189$ : "L'évaluation : contextes et pratiques », 2011/4. Cahiers internationaux de sociologie, vol. 128-129 : "Ce qu'évaluer voudrait dire », 2011. Sociologie du travail, $\mathrm{n}^{\circ} 53$ : Dossier-débat « New Public Management et professions dans l'État : au-delà des opposi- 
tions, quelles recompositions ? », 2011/3.

23. «Toute approche de l'altérité se présente sous forme relationnelle », dit Alexis Nouss («Altérité », in F. Laplantine, A. Nouss, Le Métissage, Paris, Flammarion, p. 56).

24. On devrait donc parler d'altération, terme que la langue française n'a malheureusement défini qu'en négatif. $C f$. Jean Paul Filiod, « Les arts en contexte scolaire. Le changement en mode mineur-majeur », in A. Kerlan (dir.), Des artistes à la maternelle, LyonParis, CRDP-SCÉRÉN, 2005, p. 157.

25. Alexis Nouss, op. cit., p. 59.

26. Voir par exemple des propos d'enseignantes ayant travaillé dans des écoles EAL : Sophie Buisson, «Point de vue d'une enseignante », in C. Bolze, S. Buisson, J. Desgoutte, «Artistes en résidence en maternelle », in Cahiers pédagogiques, $\mathrm{n}^{\circ} 464,2008$, p. 28. Catherine Hurtig-Delattre, "Chemin faisant... », in C. Llobet, C. Hurtig-Delattre, Les Petits Bruits, Lyon, Enfance Art et Langages, collection Embarquement immédiat, 2013, p. 6

27. Cette particularité de l'école maternelle peut donner à réfléchir sur l'éducation artistique en école élémentaire ou dans le cycle secondaire, où les acteurs n'ont la possibilité de compter, le plus souvent, que sur deux adultes pour organiser les activités. Cela peut inviter à penser, dans ces niveaux du cursus scolaire, des projets qui engagent au moins deux artistes et non un(e) seul(e).

28. Ces quatre catégories sont empruntées à Renaud Sainsaulieu (L'Identité au travail, Paris, Le Seuil, 1977), qui les a définies, en son temps, en termes d' « identité groupale ».

29. Jean-Pierre Boutinet, Anthropologie du projet, Paris, PUF, 1990, pp. 272-273.

30. Ariel Mendez (dir.), Processus. Concepts et méthode pour l'analyse temporelle en sciences sociales, Louvain-la-Neuve, Academia-Bruylant, 2010.
31. Gérald Gaglio, Sociologie de l'innovation, Paris, PUF, 2011, pp. 86-92.

32. Jean Paul Filiod, «Des malentendus, bien entendu ! Partenariat, tensions et innovations discrètes dans un dispositif de "résidence d'artistes en école maternelle" ", in Les Sciences de l'éducation. Pour l'ère nouvelle, $\mathrm{n}^{\circ} 43$ : Les partenariats institutions scolaires/ institutions culturelles, 2010/4, pp. 77-92.

33. On notera la référence à l'inspecteur, supérieur hiérarchique de l'enseignant, alors qu'on appelle coordonnateur celui des Atsem.

34. Cet exemple sur la place du massage à l'école n'est pas un épiphénomène. Quelques changements se voient ici ou là, avec l'entrée de la question du « bien-être » à l'école, dont le corps peut être un opérateur. Le yoga a d'ailleurs récemment fait l'objet d'un accord avec le ministère de l'Éducation nationale, de l'Enseignement supérieur et de la Recherche : Agrément national accordé au titre des associations éducatives complémentaires de l'enseignement public à l'association "Recherche sur le Yoga dans l'éducation (RYE) », arrêté du 4 juillet 2013. Créée en 1978, cette association a essaimé dans de nombreux pays et est invitée chaque année depuis 2014 au Congrès annuel de l'AGEEM (Association générale des enseignants des écoles et classes maternelles publiques). Nous entendions déjà lors d'un des stages d'EAL, de la bouche d'une enseignante, à propos d'une Atsem (différente de la précédente) : «Elle nous disait qu' elle utilisait parfois le yoga avant la sieste comme petit truc de relaxation, de façon assez informelle. "

35. Norbert Alter, L'Innovation ordinaire, Paris, PUF, 2000.

36. Jean Paul Filiod, «L'innovation en mode pluriel et relatif...», op. cit. 


\title{
$\mathrm{R} \cdot \mathrm{E} \cdot \mathrm{S} \cdot \mathrm{U} \cdot \mathrm{M} \cdot \mathbf{E}$ some others discretly inserted in the everyday life of « educative work ».
}

Centré sur l' « éducation artistique », cet article interroge les positionnements professionnels et la nature des engagements des différents acteurs impliqués dans des résidences d'artistes en milieu scolaire. S'appuyant sur un long travail de terrain sur le " programme de résidences d'artistes en école maternelle » Enfance Art et Langages (Lyon, 2002-2015), il s'intéresse au faire-ensemble dans des contextes de partenariat et de travail sur projet. Il montre que ces contextes impliquent les sujets individuels et collectifs concernés (enseignants, artistes, Atsem) dans un jeu de tensions entre le partenariat comme injonction institutionnelle et le partenariat comme expérience subjective. Nourrie de sociologie, d'anthropologie et de sciences de l'éducation et de la formation, l'attention portée aux expériences de ces sujets donne à voir des formes d'innovation pas toujours reconnues, voire empêchées, quand d'autres s'installent discrètement dans le quotidien du « travail éducatif ».

\begin{abstract}
This paper discusses the professional positionings and the nature of involvement when several social actors work in schools where artists intervene. Based on an extended fieldwork (Enfance Art et Langages, a program of artistic residences in pre-schools based in Lyon, created in 2002, stopped in 2015), it focusses on « doing-together » practices in partnership and projects contexts. The paper shows how these social actors (teachers, artists, teachers pre-school assistants) are involved in the partnership both considered as institutional encouragement and subjective experience. Using sociology, anthropology and educational research, the observations of the actors' experiences show innovations, some not always admitted, some stopped,
\end{abstract}


